\title{
Extranodal Rosai-Dorfman disease with bilateral orbital involvement: Report of a case treated with systemic steroid alone
}

\author{
Safiye Yilmaz' \\ Melih Ture' \\ Ahmet Maden' \\ Mine Tunakan² \\ 'Department of Ophthalmology, \\ 2Department of Pathology, Izmir \\ Atatürk Training and Research Hospital, \\ Izmir, Turkey
}

\begin{abstract}
We report the case of a 41-year-old female with bilateral orbital Rosai-Dorfman disease (RDD) and the result of corticosteroid therapy. The patient developed a swollen mass of bilateral upper and lower eye-lid over a six-month period. Diagnosis was based on the characteristic histopathologic features. She was treated with oral corticosteroids $60 \mathrm{mg} / \mathrm{per}$ day for ten days and the dose of corticostreoid was tapered every week. She is currently being treated with $10 \mathrm{mg} /$ per day for 4 months. In general, the prognosis is good, with experiencing remission following a course of oral corticosteroids. There is no evidence that corticosteroids are beneficial in the treatment of RDD in the literature, but data regarding such treatment are sparse. A more formalized surveillance of these cases would help to define more clearly the natural history of the disease and the effects of various treatment modalities.
\end{abstract}

Keywords: Rosai-Dorfman Disease, orbital involvement, treatment, corticosteroid

\section{Introduction}

Rosai-Dorfman disease (RDD) is an uncommon reactive histiocytic proliferative disorder of unknown cause (Wong et al 1989). The first case of RDD was reported in 1965, but its importance was not recognized until Rosai and Dorfman described four cases of what they called "sinus histiocytosis with massive lymphadenopathy" in 1969 (Rosai and Dorfman 1969). This is a nonneoplastic disease of unknown etiology observed mainly in adolescents with markedly enlarged cervical or other lymph nodes (Wong et al 1989). RDD generally presents third to fifth decades of life (although presentation in children and the elderly has also been reported) with cervical lymphadenopathy often preceded by symptoms suggestive of a viral infection (Foucar et al 1979). The majority of patients have elevation of the erythrocyte sedimentation rate (ESR) and a polyclonal increase in globulins (Yip et al 2002). Nevertheless, there are numerous published cases without lymphadenopathy. So-called extranodal disease occurs in some $25 \%$ of cases and may arise in the orbit, nasopharynx, respiratory pathways, gastrointestinal tract, endocrine glands (particularly the thyroid), bone, skin, and nervous system. Ten percent of patients have orbital involvement, but only one-third of those with orbital involvement have bilateral disease. The disease is usually chronic and refractory to various treatment modalities.

There are previously reported cases of ocular RDD without clinically detectable systemic involvement, however we report the clinical and histopathological features of a case of RDD with bilateral orbital and unusual articular, abdominal involvement, and the successfull result of systemic streoid treatment.

\section{Case report}

The patient was a 41-year old woman, who developed a swollen mass of bilateral upper and lower eye-lid over a six-month period (Figure 1a). Examination revealed 


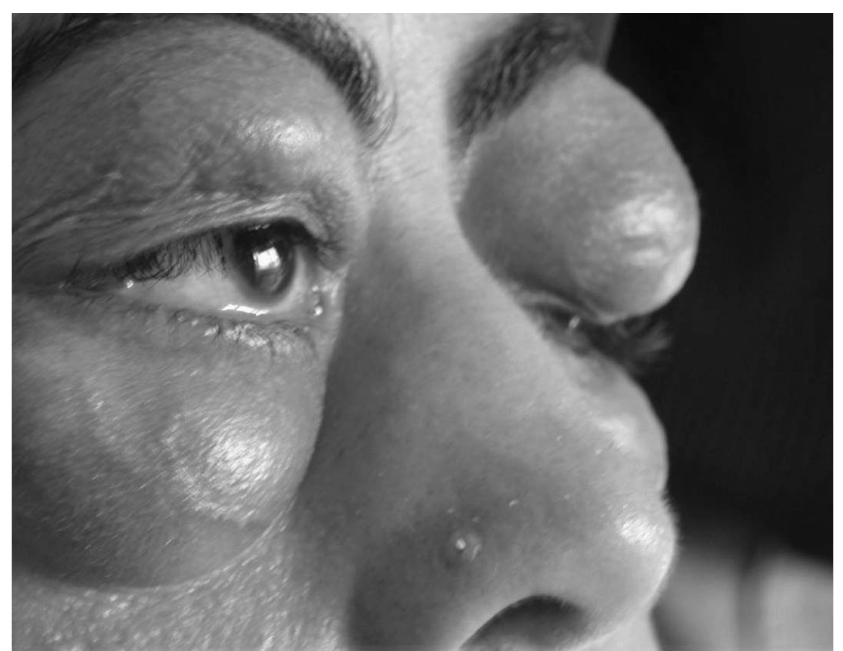

Figure I Pre-treatment photography of the patient shows a swollen mass of bilateral upper and lower eye-lid over a six-month period.

a large, noninflamed, nontender mass in bilateral lower and upper eyelid. Anterior segment examination and fundoscopy were essentially normal. There was no preauricular or cervical lymph node enlargement. On systemic evaluation, there were severe congenital deformations both on hands and legs which couldn't be related to any rheumatological or other systemic disease. She also had poor hearing since early childhood which diagnosed as bilateral sensorio-neural hearing loss. Also in her history, 17 years ago, there was an abdominal surgery with poor medical records.

Coronal images obtained by magnetic resonance imaging showed a homogeneous mass involving bilateral infratemporal fossa, nasale cavity, lacrimal gland, and buccal and perimandibular interface in the left side (Figure 2). Surgical excision biopsy of the mass was performed under local anesthesia. Histopathological examination was consistent with a diagnosis of RDD. The infiltrate is composed of histiocytes with a characteristic immunohistochemical profile (S100-positive) together with small lymphocytes and plasma cells.

She was treated with oral corticosteroids $60 \mathrm{mg} /$ per day for ten days and the dose of corticostreoid was tapered every week. She is currently being treated with $10 \mathrm{mg} /$ per day for 6 months.

In general, the prognosis is good, with the remission of orbital masses following a course of oral corticosteroids at one month (Figure 3).

\section{Conclusion}

Ocular involvement in RDD is rare (\%10) (Foucar et al 1979). The diagnosis of RDD was based on histopathological

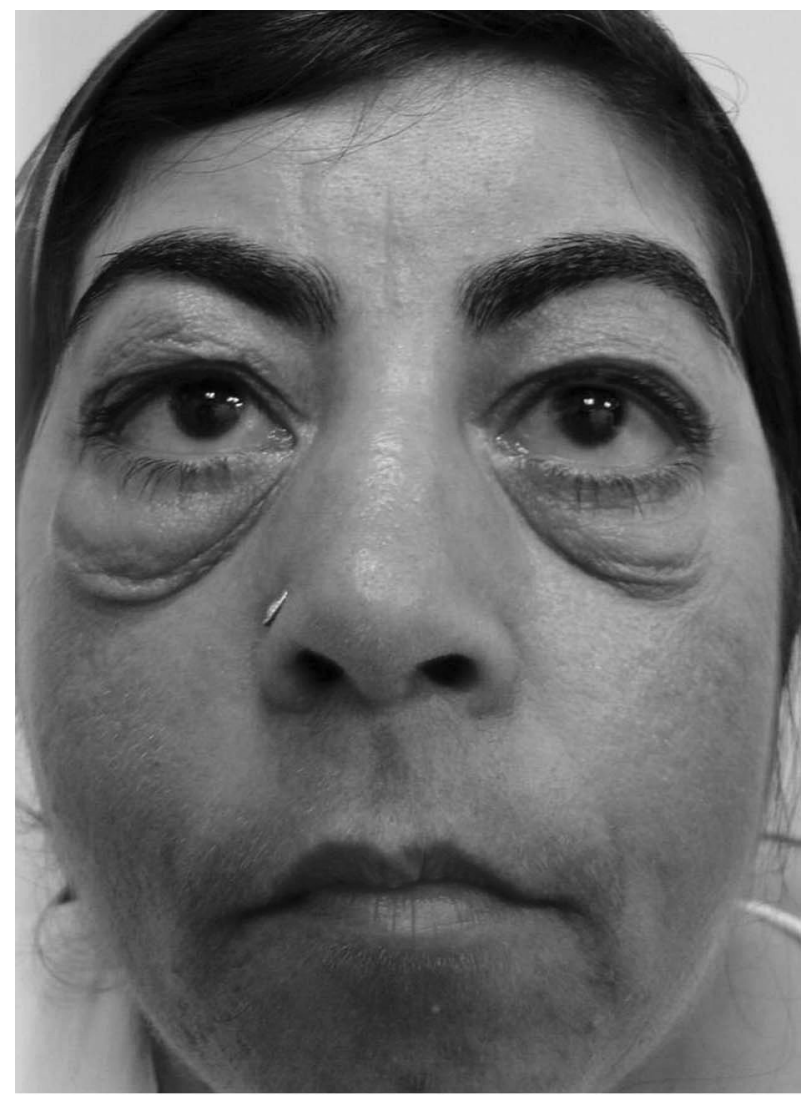

Figure 2 Post-treatment photography shows an partial remission of orbital masses following a course of oral corticosteroids at one month.

examination with very clear and typical histopathological features of the disease described above.

However, there was no previous report and no relationship, as far as we know, between the mass lesions of RDD and either the deformities on extremities or the hearing loss or history of the abdominal surgery. A previous report was mentioned about a patient of RDD with bilateral orbital masses having subcutaneous masses in the lower abdominal wall, backache and lower extremity weakness. They reported
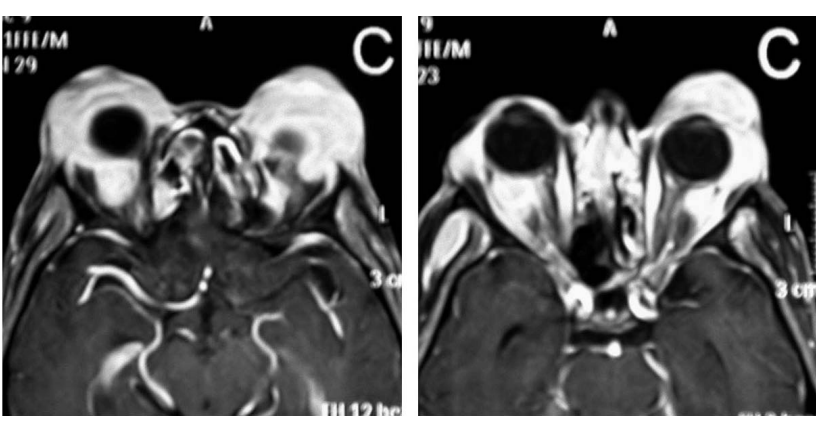

Figure 3 Coronal images obtained by magnetic resonance imaging showed a homogeneous mass involving bilateral infratemporal fossa, nasale cavity, lacrimal gland, and buccal and perimandibular interface in the left side. 
that the pathologic specimens from the abdominal masses, paravertebral tissues, and eyelid tissues were reviewed, and the final diagnosis was RDD (Yip et al 2002). So, we don't know if these separate findings in our cases were coincidental or any unknown feature of the disease. Our systemic work-up results were negatively for RDD.

The data available do not allow for the establishment of definitive treatment protocols for the ocular complications of RDD, although many therapeutic options, such as surgery, radiotherapy, oral corticosteroids, and antibiotics, have been described (de Silva and Joshi 2005). Reports of complete remission are few and most have occurred with the use of combinations of corticosteroids, vinca alkaloids, and alkylating agents (Komp 1990). Treatment is indicated if there is functional impairment of one or more organ systems (Mohadjer et al 2006). We used oral corticosteroid alone such a patient of RDD with eyelid masses. She was treated with oral corticosteroids $60 \mathrm{mg} /$ per day for ten days and the dose of corticostreoid was tapered at $10 \mathrm{mg}$ every week. She is currently being treated with $10 \mathrm{mg} / \mathrm{per}$ day for 6 months. In general, the prognosis is good, with experiencing remission of orbital masses following a short course of oral corticosteroids. A positive response to the treatment was seen in the first week. The stabilization was achieved in one month.

RDD is a rare condition and should be considered in the differential diagnosis of orbital or eyelid soft tissue enlargement. Medical management with corticosteroid should be considered, as RDD was diagnosed. Long-term follow-up is required as potentially vision-threatening complication and recurrence may occur.

\section{References}

de Silva D, Joshi N. 2005. Rosai-Dorfman disease recurrence with bilateral orbital masses following immunosuppressant therapy. Orbit, 24:51-3.

Foucar E, Rosai J, Dorfman RF. 1979. The ophthalmologic manifestations of sinus histiocytosis with massive lymphadenopathy. Am J Ophthalmol, 87:354-67.

Komp DM. 1990. The treatment of sinus histiocytosis with massive lymphadenopathy (Rosai-Dorfman disease). Semin Diagn Pathol, 7:83-6.

Mohadjer Y, Holds JB, Rootman J, et al. 2006. The spectrum of orbital Rosai-Dorfman disease. Ophthal Plast Reconstr Surg, 22:163-8.

Rosai J, Dorfman RF. 1969. Sinus histiocytosis with massive lymphadenopathy: a newly recognized benign clinicopathologic entity. Arch Pathol, 87:63-70.

Wong HC, Wright JE, Harry J. 1989. Rosai-Dorfman disease (sinus histiocytosis with massive lymphadenopathy) with cutaneous and ocular involvement: a case report. Eye, 3:849-53.

Yip CC, Cheng CL, Poh WT, et al. 2002. Orbital, adnexal, and unusual systemic involvement in Rosai-Dorfman disease. Ophthal Plast Reconstr Surg, 18:223-7. 
